References:

[1] Romero-Pozuelo, J. et al. (2014) Journal of Cell Science 127, 4246-4259.

[2] Baños-Mateos, S. et al. (2014) Acta Crystallographica A70, 530-534.

[3] Mansilla, A. et al. (2017) PNAS 114(6), E999-E1008.

Keywords: calcium sensor, molecular recognition, proteinprotein interaction
MS10-02

\section{Selective protonation and stereoelectronic effects govern catalysis in a pyridoxal-5'- phosphate-dependent enzyme}

Andrey Kovalevsky ${ }^{1}$

1. Neutron Scattering Division, Oak Ridge National Laboratory, Oak Ridge, United States of America

email: kovalevskyay@ornl.gov

Pyridoxal-5' -phosphate (PLP, vitamin $\mathrm{B}_{6}$ derivative) is one of the most ubiquitous co-factors in biological systems. At least 140 different types of biochemical transformations are catalyzed by PLP-dependent enzymes, comprising $4 \%$ of all classified enzymatic activity. PLP-dependent enzymes perform some of the most difficult chemical reactions involving amines and amino acids, including transamination, racemization, phosphorylation, decarboxylation, aldol cleavage, elimination, and replacement reactions. PLP-dependent enzymes are categorized into five recognized fold-types (I-V), each performing characteristic chemistry. Despite decades of research, the physical determinants that govern specificity of and catalytic enhancement by PLP-dependent enzymes remain largely unresolved. Fold-type I is the most prevalent, mainly promoting transamination and decarboxylation reactions. Two major hypotheses have been developed to understand the different types of chemistry in PLP-dependent enzymes, namely 1) stereoelectronic control and 2) electronic modulation through selective protonation. An enzyme active site local environment can promote different protonation profiles of PLP during the catalytic cycle. We report the first neutron crystal structure of a Fold I PLP-dependent enzyme, aspartate aminotransferase (AAT). In crystal, AAT was captured in both the internal and external aldimine states after soaking with substrate analog a-methyl-D-aspartic acid. The neutron structure illustrates the protonation state differences between the reactant (internal aldimine) and intermediate (external aldimine) states, and sheds new light on the catalytic mechanism of transamination. We are using QM/MM calculations and MD simulations based on the correct models derived from the neutron structure, and neutron vibrational spectroscopy for deeper understanding of the enzyme mechanism and to uncover the contribution of low-energy protein vibrations to catalysis.

Keywords: protonation, catalysis, dynamics 